\title{
Subjective Beliefs, Memory and Functional Health: Change and Associations over 12 Years in the Australian Longitudinal Study of Ageing
}

\author{
Mary A. Luszcz ${ }^{a}$ Kaarin J. Anstey ${ }^{b}$ Paolo Ghisletta ${ }^{c, d}$ \\ ${ }^{a}$ Flinders Centre for Ageing Studies, School of Psychology, Flinders University, Adelaide, S.A., and ${ }^{b}$ Centre for \\ Research on Ageing, Health and Wellbeing, Australian National University, Canberra, A.C.T., Australia; ${ }^{\circ}$ Faculty of \\ Psychology and Educational Sciences, University of Geneva, Geneva, and d Distance Learning University, Switzerland
}

\section{Key Words}

Beliefs · Memory $\cdot$ Health $\cdot$ Longitudinal changes

\begin{abstract}
Background: Neither subjective memory beliefs, nor remembering itself, can be isolated from the overall context in which one is aging, nor are the drivers of memory complaints well specified. Sense of control is an important self-regulatory resource that drives cognitive and physical health over the lifespan. Existing findings are equivocal concerning both the extent of stability or change in control beliefs over time as well as their contribution to changes in behavior. Objective: Subjective beliefs may play a role when engaging memory processes or identifying memory complaints, and it has been argued that self-regulatory potential in general may be limited by age-related changes in the domains of health and cognition. We aimed to examine trajectories of change and shed light on relationships among subjective beliefs and indicators of memory and functional health. Methods: Participants' data were drawn from four measurement occasions over up to a 12-year period (1992-2004) from the Australian Longitudinal Study of Ageing (ALSA), a population-based study of older adults [age 65-100 years; mean age(SD) at the
\end{abstract}

first and final occasion 78.2 (6.7) and 84.9 (4.9) years, respectively]. Participants completed three questionnaires assessing subjective beliefs concerning (1) memory knowledge and control, (2) health control, and (3) expectancy of control over a range of lifestyle situations. Memory comprised a recall composite. Functional health tapped mobility and disability. Latent growth curve models incorporated informative covariates (baseline age, gender, self-rated health, education, and chronic conditions). Results: While subjective memory control beliefs, but not subjective knowledge of memory tasks, improved over 12 years, neither was associated with level of memory performance. Knowledge of memory tasks was linked to a significant memory decline. Beliefs about memory, health, and lifestyle were interrelated. Declines in remembering and health were also coupled; moreover, changes in both were coupled with change in lifestyle control beliefs. Conclusions: This is the first examination of individual differences in changes in, and relationships among, psychological domains of subjective beliefs about memory, health, and lifestyle, and objective remembering and functional health in very late life. Findings point to a system of coupled changes in memory and health in late life that is related to underlying beliefs about control over lifestyle.

(c) 2015 S. Karger AG, Basel

\section{KARGER 125}

(c) 2015 S. Karger AG, Basel

0304-324X/15/0613-0241\$39.50/0

E-Mail karger@karger.com

www.karger.com/ger
Prof. Mary A. Luszcz

Flinders Centre for Ageing Studies

School of Psychology, Flinders University

GPO Box 2100, Adelaide, SA 5001 (Australia)

E-Mail mary.luszcz@ flinders.edu.au 
Subjective memory complaints (SMCs) comprise one of a multifaceted range of beliefs about memory ability and may become more frequent with advancing age [1]. Interrelations among such beliefs may comprise a complex interactive self-regulatory system that underlies memory complaints and performance. Drivers of memory complaints are not well known, nor can SMCs or remembering itself be isolated from the overall context in which aging occurs $[2,3]$. Conceptually, it is becoming increasingly evident that physical and cognitive functioning are interdependent [4-6], sharing common variance [7]. Subjective beliefs, particularly about control or selfefficacy, may play a self-regulatory role when engaging memory processes [5]. Moreover, self-regulatory potential in general may be limited by age-related changes in the domains of health and cognition [8], as being mobile and retaining one's memory are two hallmarks of aging well.

The prominence of concerns about declines in these arenas and how they are linked to personal control beliefs and knowledge of the memory system may extend the understanding of SMC. Of central interest is the possibility that levels of, and change in, generic perceived control, knowledge of the memory system, and domain-specific perceived control over memory and/or health are associated with levels of, or aging-related changes in, memory and/or functional health (FHlth). We know very little about possible couplings of, or changes in, this constellation of domains. Our aim is to take a step back from SMCs per se and examine memory by including potential upstream factors, such as perceived control, knowledge of memory, and broader systemic deterioration, e.g., in FHlth, that may be associated with them.

We sought behavioral indicators of cognition and health [9]. Episodic memory provides one performance criterion. For health, we targeted function related to mobility and disability. We adjusted for health status, rather than targeting health per se, because the consequences for similar health indicators vary widely among individuals.

Control beliefs can be broadly defined as the subjective perceptions of the extent to which desired outcomes can be achieved through our actions or abilities $[5,10]$. When the salience of outcomes becomes prominent, e.g., as evidenced by pervasive declines in older adults' memory and FHlth, perceived control may be especially important [11]. Given this, reductions in competence in these domains may be associated with diminished perceived control over them. By extension, memory complaints may become more common.

Knowledge of the memory system may also contribute to SMCs if a disconnect is perceived between what some- one knows to be the case about remembering and their performance, which falls short of it. Moreover, in late life, a negative relationship between memory self-efficacy and memory knowledge $[12,13]$ may highlight a sense of the limitations of oneself as a memorizer, engendering SMCs [1].

Theoretically, we draw on life-span notions of multidimensionality, multidirectionality, and contextualism [14]. Multidimensionality is reflected in the examination of generic and domain-specific perceived control beliefs, knowledge of memory, and two performance parameters. The longitudinal nature of the study enables us to look at the multidirectionality of several dimensions changing at different rates or following a variety of intraindividual patterns. We especially seek to identify coupled trajectories of change. The importance of context $[3,15]$ will emerge by controlling for a range of informative distal covariates known to be linked to control, memory, and/ or FHlth. It is well known that both memory and FHlth decline with age [9]. However, very little is known about how beliefs change over time. Nor have late-life longitudinal changes in memory and health beliefs and performance been studied together.

The use of a domain-specific measure of control may provide insights into multidimensional aspects of development that are not provided by more global measures [16]. Domain-specific approaches are useful as aging-related changes in perceptions of control vary according to domain [17]. For memory [12] and health [18], scales have been developed to capture the nuances of beliefs about these two domains. In striving for a sense of personality coherence, domain-specific control beliefs may be encompassed in a broader system in which they are closely aligned [1, 19, 20]. By examining associations among domain-specific and generic beliefs, this possibility can be assessed.

It is becoming widely accepted that beliefs and perceptions about capacity impact performance [2]. Generic perceptions of control reliably predict cognition, health, and wellbeing [21]. Australian [22] and US [5] data have linked general control to a 4 -year decline in immediate recall. Research concerned with memory self-efficacy and objective cognitive test performance also reveals positive associations [23].

Likewise, research concerned with general and healthspecific control beliefs has revealed associations with health-related outcomes. General control beliefs have protected against 9-year mortality in the Australian Longitudinal Study of Ageing (ALSA) [24] and have been associated with reduced likelihood of 14-year disability and 
Table 1. Descriptive statistics for key variables by occasion of measurement (W)

\begin{tabular}{|c|c|c|c|c|}
\hline & $\mathrm{W} 1$ & W3 & W6 & W7 \\
\hline \multicolumn{5}{|l|}{ Covariates } \\
\hline Age, years & $78.2(6.7)$ & $79.6(6.5)$ & $83.5(5.6)$ & $84.9(4.9)$ \\
\hline Male sex & 50.6 & & & \\
\hline $\mathrm{SRH}$ & $3.1(1.2)$ & $3.0(1.1)$ & $3.1(1.1)$ & $3.1(0.9)$ \\
\hline Education $>14$ years & 44 & & & \\
\hline Illnesses & $5.3(3.0)$ & $4.1(2.5)$ & $4.7(2.5)$ & $5.3(3.3)$ \\
\hline \multicolumn{5}{|l|}{ Belief measures } \\
\hline MIALOc $^{\mathrm{a}}$ & $28.42(2.84)$ & - & $30.17(3.40)$ & $30.24(3.47)$ \\
\hline MIATas $^{\mathrm{a}}$ & $61.26(5.36)$ & - & $60.28(5.89)$ & $60.40(5.36)$ \\
\hline HlthLoc ${ }^{b}$ & $15.39(3.62)$ & $14.68(3.69)$ & $15.10(3.47)$ & $15.22(3.53)$ \\
\hline GenLoc $^{\mathrm{b}}$ & $26.60(5.21)$ & $25.82(5.71)$ & $26.69(5.65)$ & $26.77(5.37)$ \\
\hline \multicolumn{5}{|l|}{ Behavioral measures } \\
\hline FHlth $^{\mathrm{b}}$ & $6.76(7.28)$ & $7.72(7.73)$ & $9.11(8.45)$ & $9.65(8.20)$ \\
\hline Items recalled & $2.26(0.95)$ & $2.39(0.88)$ & $2.43(0.70)$ & $1.88(1.05)$ \\
\hline Symbols recalled & $6.19(2.06)$ & $6.33(2.04)$ & $6.29(2.14)$ & $5.67(2.53)$ \\
\hline Pictures recalled & $5.48(2.43)$ & $5.09(2.45)$ & $5.38(2.58)$ & $4.98(2.65)$ \\
\hline \multicolumn{5}{|c|}{ 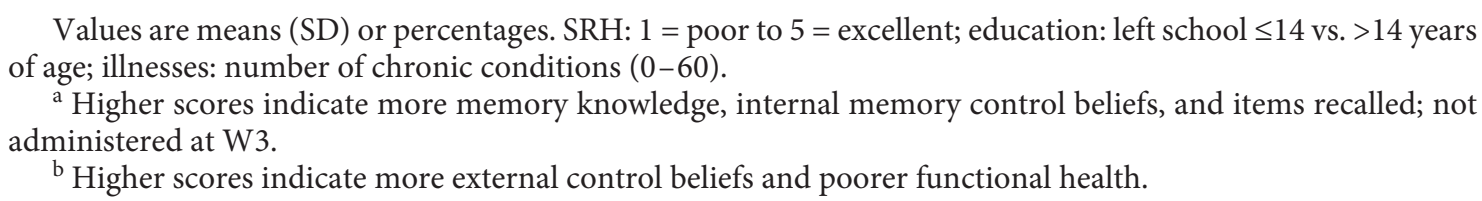 } \\
\hline
\end{tabular}

mortality in Germany [25]. Domain-specific health control beliefs also have been associated with greater engagement in health behaviors as well as better health status and mortality [10].

Existing data are equivocal concerning the extent of stability or change in perceptions of general control. There is evidence showing stability [26], decline [27], and a mixture of both [16]. Moreover, substantial individual differences in control beliefs prevail [28]. The extent to which changes in generic or domain-specific control beliefs occur or covary in later life has been neglected [10].

Informative covariates known to be linked to control, memory, or physical functioning include gender, education, health status, and age. Female gender and poorer health have been linked to reduced control beliefs [29]. Higher education and female gender both have been linked to better memory [7]. Physical function also varies between men and women. We adjust for age as both memory and FHlth decline with age $[6,9]$, and advanced age is linked to reduced subjective control within and across domains [30].

We examine average levels of, 12-year longitudinal changes in, and associations among (1) control beliefs in domains of (a) memory, (b) health and (c) lifestyle; (2) memory knowledge; (3) FHlth, and (4) memory performance, taking into account informative covariates. Specifically, we examine (a) the extent to which the stable components of the domains covary with each other, and (b) whether changes in domains are concomitant and linearly related. It is hypothesized that generic control will be linked to both FHlth and memory. For domain-specific measures, we expect stronger links within, than across, domains. A paucity of existing data precludes specific hypotheses about relationships among change.

\section{Methods}

Sample and Participant Selection

Residents over the age of 70 years were identified using the South Australian Electoral Roll. Of the 2,703 eligible individuals identified, $55 \%(n=1,477)$ agreed to participate (the baseline sample of 2,087 also included spouses and co-residents). Comparability of sample patterns of Medicare use and the proportion of older adults in residential care to the Australian population suggest representativeness [31]. Descriptive statistics are shown in table 1.

The sample was drawn from four measurement occasions over up to 12 years (1992-2004) from the ALSA. The ALSA has been described in depth [32] and here only elements pertinent to this study will be presented. Healthy participants were included 
if they had completed both the self-evaluated belief questionnaires and the memory tasks on at least one of the measurement occasions. These occurred as follows: baseline (1992-1993; $\mathrm{n}=$ $2,087)$, wave 3 (W3; 1994-1995; $\mathrm{n}=1,679)$, W6 $(2000-2001 ; \mathrm{n}=$ 779), and W7 (2003-2004; $\mathrm{n}=486)$. Attrition is mainly attributable to death.

\section{Assessments and Measures}

Belief Measures

Memory beliefs were marked by two subscales from the Metamemory in Adulthood scale [33]: Locus (MIALoc; Cronbach's $\alpha=0.72$ ) and Task (MIATas; $\alpha=0.74$ ). These measure control over and knowledge of one's own memory, respectively. Higher summed scores indicate a more internal sense of control over memory and greater memory knowledge. Health control beliefs were measured using the Internal Health Locus of Control (HlthLoc; $\alpha=0.74$ ) subscale from the Multidimensional Health Locus of Control Scale [18]. Six items were answered on a Likert scale from strongly agree (1) to strongly disagree (5). Lower summed scores indicate more internality. Lifestyle or generic sense of control (GenLoc; $\alpha=0.71$ ) was measured by the Expectancy of Control subscale of the Desired Control Measure [34], designed specifically for older adults. The 12 items ask respondents to indicate the extent to which they believe they have control in areas of involvement with others and engagement in activities. Items were answered on a Likert scale from strongly agree (1) to strongly disagree (5), with lower summed scores indicating a more internal expectancy of control.

\section{Behavior Measures}

Memory. Recall (memory) was measured using three tasks: items recalled during the Mini-Mental State Examination, symbols recalled after completion of the Digit-Symbol Substitution Subscale of the WAIS, and pictures recalled after completion of a 15item version of the Boston Naming Task [15]. Memory is the only variable defined as a latent measure [7]; higher scores indicate better memory.

Functional Health. FHlth $(\alpha=0.85)$ was used as a marker of physical health status. Specifically, mobility [35] and disability [36] were assessed. Responses to the latter items were made on Likert scales: (0) no difficulty, (1) a little difficulty, (2) some difficulty, (3) a lot of difficulty, and (4) just unable to do it; dichotomous responses were recoded to the extremes of this scale. Higher scores indicate worse FHlth (range $=0-28$ ).

Covariates. Among informative covariates were participants' baseline age (years), gender $(0=$ male; $1=$ female $)$, self-rated health (SRH; $1=$ poor to $5=$ excellent), education level (left school at $\leq 14$ vs. $>14$ years of age), and number of current and previous chronic conditions (illnesses: 0-60).

\section{Analysis Strategy}

The analyses used the Full Information Maximum Likelihood (FIML) algorithm, which is among the most powerful statistical treatments of incomplete data [37]. FIML unbiases parameter estimates by including covariates informative of the data incompleteness mechanism. FIML permits analysis of data of all persons, including those not assessed at all time points or on every measure at a given occasion, and adjusts for design-based data unbalance (i.e., memory measured at W1, W3, W6, and W7, but MIALoc and MIATas at W1, W6, and W7).
We applied latent curve models (LCM) $[38,39]$ over four occasions, implemented with Mplus 6.12 [40]. The simplest specification of the LCM presumes that a variable $Y$, for a given individual at a given time point, changes linearly as a function of time. Figure 1a depicts a linear LCM adapted to our data (W1, W3, W6, and W7 of ALSA). Of particular interest are the parameters associated to level (L), which represents the general level of performance in $\mathrm{Y}$, and change (C), which assesses how Y changes linearly in time. For both level and change, the model estimates an average sample value $\left(M_{L}\right.$ and $M_{C}$ in fig. 1a), which represents the overall sample's tendency, as well as the variance $\left(V_{L}\right.$ and $V_{C}$ in fig. 1a), which defines the extent to which individuals' values deviate from the sample average value. The covariance between level and change is also estimated $\left(\mathrm{C}_{\mathrm{LC}}\right.$ in fig. 1a), and informs about the relationship between level and change. To unbias estimates about level and change due to incomplete data, we used the FIML algorithm and added the five time-invariant covariates as predictors of variance in the level and, if present, the change (for simplicity, covariates are not represented in fig. 1a). Any remaining deviation between the observed and the predicted value of $\mathrm{Y}$ is captured by the uniqueness component $\mathrm{U}$, whose variance is assumed constant in time (at $\left.\mathrm{V}_{\mathrm{U}}\right)$.

We first applied the LCM to each variable separately (i.e., in a univariate fashion, as represented in fig. 1a, for MIALoc, MIATas, HlthLoc, GenLoc, FHlth, and memory) to ascertain the most appropriate specification of each univariate model [7]. In particular, we tested whether the variance in change $\left(V_{C}\right)$, representing interindividual differences in intraindividual change, was significant. Second, all variables were merged in a comprehensive multivariate LCM (MLCM), where level and change components (L and C) were allowed to correlate. Figure $1 \mathrm{~b}$ represents the structural part (only the latent variables, hence levels and changes) of the MLCM. All the levels were allowed to intercorrelate (represented by the continuous two-headed arrows). The changes with significant variance were also allowed to intercorrelate (dashed two-headed arrows), and to correlate with their respective level (dotted twoheaded arrows). Time was defined as the average amount of time in study since baseline. To account for age heterogeneity, we included participants' age among the covariates.

\section{Results}

The series of univariate LCMs made it possible to ascertain the best LCM specification of each variable independently. This first analytical step is important when the final aim is to define a MLCM to describe relations in level and change among multiple variables. Because both means and variances are residualized for the effects of the covariates, the estimates correspond to the residual variance that is left after their effects are removed.

Results showed that all univariate models adjusted well to the data (average values for goodness of fit indices were 0.954 CFI, 0.028 RMSEA, and 0.045 SRMR; estimates are not shown due to space limitations but are available from M.A.L. on request). Means and variances in level were significant in all variables. The mean change was different 


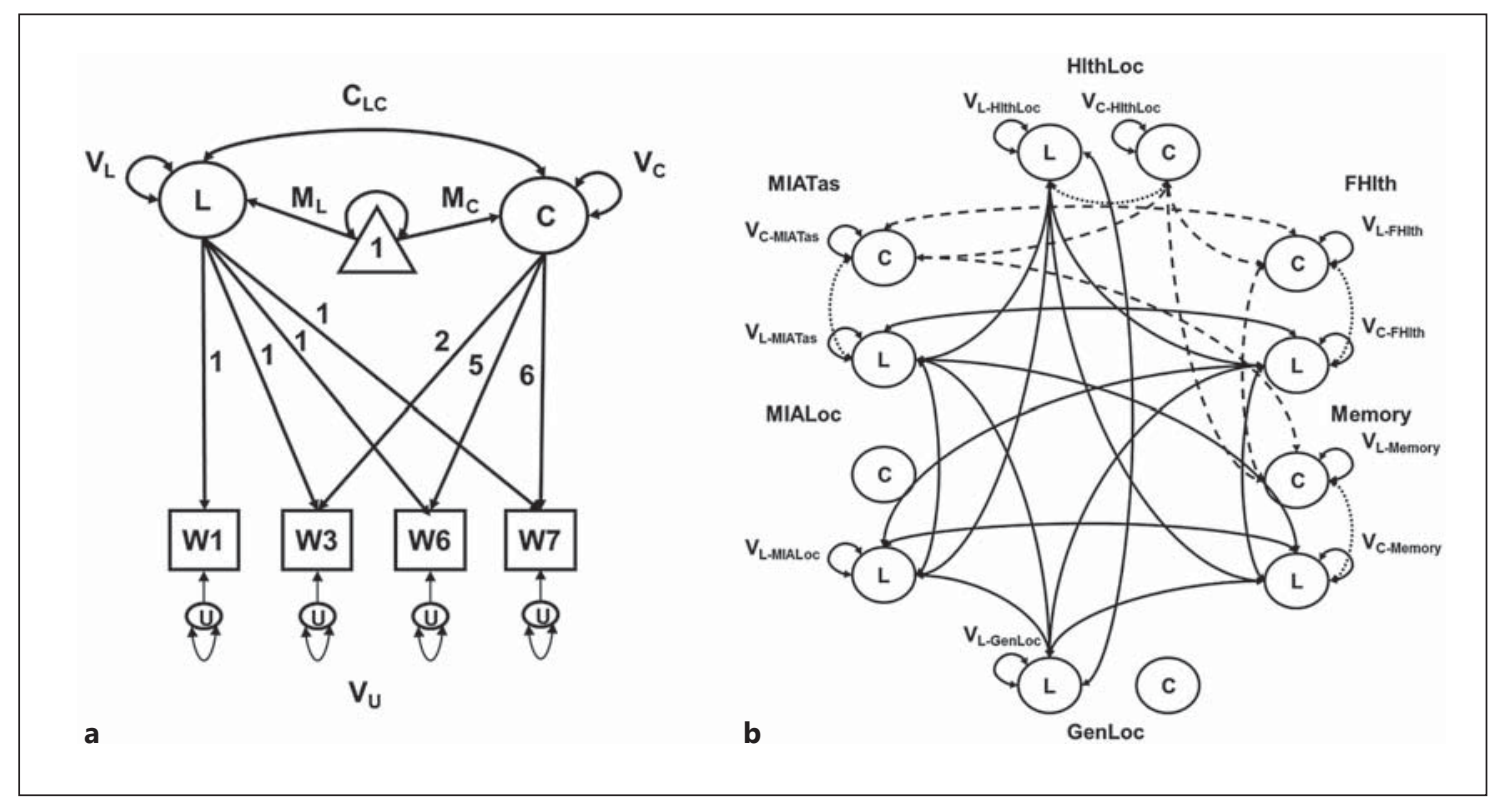

Fig. 1. a Representation of a univariate LCM. Squares represent manifest variables (assessments at W1, W3, W6, and W7), circles represent the latent variables level $(\mathrm{L})$ and change $(\mathrm{C})$, and the triangle is inserted to represent the estimation of means (for level and change, $\mathrm{M}_{\mathrm{L}}$ and $\mathrm{M}_{\mathrm{C}}$, respectively). One-headed arrows represent regression weights (or factor loadings), and two-headed arrows represent variances and covariances. b Representation of the structural part (levels and changes) of a MLCM. Represented are covariances among levels (continuous two-headed arrows), covariances among changes with significant variance (dashed twoheaded arrows), and covariances among level and change with significant variance of the same construct (dotted two-headed arrows). For simplicity, covariances among levels and changes with significant variance of different constructs were omitted. from zero in all variables except MIATas and HlthLoc. There was variability in change in all variables except MIALoc and GenLoc. This implies that change in these two constructs was the same (i.e., constant) across individuals and hence change could neither covary with other variables, nor be predicted by external covariates.

Second, we ran the MLCM, bearing in mind results of the univariate models, especially for MIALoc and GenLoc. The MLCM fit the data quite well $\left[\chi^{2}{ }_{398}=\right.$ $1,096,330$, RMSEA $=0.029(90 \% \mathrm{CI}=0.027-0.031), \mathrm{CFI}=$ 0.916 , and SRMR $=0.145]$. Growth parameter estimates, virtually identical to those from the univariate LCMs, are shown in the last two columns in table 2. Thus, the higher-dimensional estimation procedure worked well despite the much larger number of variables; the only mean changes not different from zero were again MIATas and HlthLoc. The residual change variances of MIALoc and GenLoc were again not significant. Consequently, the correlations involving the changes of MIALoc and GenLoc are not defined.

Focusing on the change parameters, results indicated that MIALoc showed an average increase over time (i.e., increased perceptions of internal control over memory). Significant interindividual variability in rates of change was evident for MIATas and HlthLoc, despite their mean changes not being significant. GenLoc showed an average increase, indicating a shift to more external generic perceptions of control, but no significant variance in change. Parameters for the performance measures indicated average deterioration in memory and FHlth, although significant change variances pointed to heterogeneity in rates of change for both.

The real advantage of the MLCM is the estimation of the linear associations among the growth parameters of each variable (table 2 ). That is, the model estimated covariances that define the interrelationships separately for levels (top panel), change (middle panel) and levels with changes (bottom panel) for belief (excluding MIALoc and HlthLoc) and function variables.

For levels, MIATas and MIALoc were correlated negatively with each other and with HlthLoc. GenLoc correlated positively with MIALoc, HlthLoc and FHlth and negatively with memory. For changes, the heterogeneous patterns of change in MIATas were negatively related to 
Table 2. Growth parameter estimates and standardized covariances (correlations) within (top two panels) and between (bottom panel) levels and changes in MLCM, net of covariates

\begin{tabular}{|c|c|c|c|c|c|c|c|c|}
\hline & \multirow[t]{2}{*}{ MIATas $^{\mathrm{a}}$} & \multirow[t]{2}{*}{ MIALoc $^{\mathrm{a}}$} & \multirow[t]{2}{*}{ HlthLoc ${ }^{b}$} & \multirow[t]{2}{*}{ GenLoc $^{\mathrm{b}}$} & \multirow[t]{2}{*}{ FHlth $^{\mathrm{b}}$} & \multirow[t]{2}{*}{ Memory $^{\mathrm{a}}$} & \multicolumn{2}{|c|}{ Growth parameter estimates } \\
\hline & & & & & & & means & variances \\
\hline \multicolumn{9}{|l|}{ Levels } \\
\hline MIATas & & & & & & & $61.495^{*}$ & $13.961^{*}$ \\
\hline MIALoc & $-0.792^{*}$ & & & & & & $28.405^{*}$ & $1.881^{*}$ \\
\hline HlthLoc & $-0.293^{*}$ & $-0.685^{*}$ & & & & & $14.918^{*}$ & $7.890^{*}$ \\
\hline GenLoc & -0.282 & $0.142^{*}$ & $0.142^{*}$ & & & & $27.086^{*}$ & $14.960^{*}$ \\
\hline FHlth & 0.001 & 0.029 & 0.016 & $0.127^{*}$ & & & $5.425^{*}$ & $24.213^{*}$ \\
\hline Memory & 0.056 & -0.082 & 0.034 & $-0.191^{*}$ & $-0.097^{*}$ & & $5.825^{*}$ & $1.609^{*}$ \\
\hline \multicolumn{9}{|l|}{ Changes ${ }^{\mathrm{c}}$} \\
\hline MIATas & & & & & & & -0.104 & $0.283^{*}$ \\
\hline MIALoc & - & & & & & & $0.227^{*}$ & 0.029 \\
\hline HlthLoc & $-0.450^{*}$ & - & & & & & -0.001 & $0.050^{*}$ \\
\hline GenLoc & - & - & - & & & & $0.235^{*}$ & 0.027 \\
\hline FHlth & $0.446^{*}$ & - & -0.022 & - & & & $0.691^{*}$ & $0.316^{*}$ \\
\hline Memory & $-0.348^{*}$ & - & 0.136 & - & $-0.420^{*}$ & & $-0.140^{*}$ & $0.015^{*}$ \\
\hline \multicolumn{9}{|c|}{ Level vs. changec } \\
\hline MIATas & 0.060 & $-0.513^{*}$ & 0.109 & 0.094 & -0.060 & $-0.530^{*}$ & & \\
\hline HlthLoc & 0.165 & $0.329^{*}$ & $-0.358^{*}$ & $-0.181^{*}$ & 0.138 & 0.142 & & \\
\hline FHlth & 0.066 & 0.093 & 0.018 & 0.042 & $-0.130 *$ & $-0.333^{*}$ & & \\
\hline Memory & -0.137 & -0.081 & -0.005 & -0.051 & 0.088 & $0.186^{*}$ & & \\
\hline
\end{tabular}

Covariates: sex: $(0=$ male, $1=$ female), SRH $(1=$ poor to $5=$ excellent $)$, education level (left school $\leq 14$ vs. $>14$ years of age $)$ and number of chronic conditions $(0-60)$. Bold entries in the bottom panel are level-change correlations of the same variables. $* \mathrm{p}<0.01$.

${ }^{\text {a }}$ Higher scores indicate more memory knowledge, internal memory control beliefs, and items recalled.

${ }^{\mathrm{b}}$ Higher scores indicate more external control beliefs and poorer functional health.

${ }^{\mathrm{c}}$ MIALoc and GenLoc values are not shown due to nonsignificant residual change variance.

memory and HlthLoc changes, but positively to FHlth change. Change in memory was correlated negatively with FHlth. Correlations of levels and changes showed that levels of MIALoc correlated negatively with change in MIATas and positively with change in HlthLoc, which also correlated negatively with levels of GenLoc. Levels and changes intercorrelated negatively for HlthLoc and FHlth. Finally, level of memory correlated negatively with change in MIATas and FHlth and positively with change in memory.

Table 3 summarizes the effects of the covariates on level and change for each variable. For levels, age positively influenced GenLoc (more external) and FHlth (more illnesses) and negatively influenced memory (worse). Being female was associated to higher levels of MIALoc (internal), FHlth, and memory. Better SRH negatively affected HlthLoc (more internal), GenLoc, and FHlth (fewer illnesses) and positively affected memory. More education linked with external HlthLoc and better memory and fewer illnesses (FHlth). Finally, the number of illnesses nega- tively affected MIALoc and positively affected HlthLoc, FHlth, and memory. With respect to the changes, age positively influenced FHlth and negatively influenced memory (i.e., both erode with aging); being female was associated to less decline in GenLoc; poorer SRH and more illnesses were associated with less change in memory knowledge (MIATas) and FHlth; more illnesses related to increasingly external GenLoc, and more education positively influenced change in MIATas and FHlth.

\section{Discussion}

Sense of control is an important self-regulatory resource that drives cognitive and physical health over the lifespan, although the potential for self-regulation may be limited by age-related changes in these domains. It could be argued that in the realm of subjective assessments of memory, complaints of poor memory may arise if people sense that they no longer have control over their 
Table 3. Covariate effects in MLCM

\begin{tabular}{lcccccc}
\hline Covariates & MIATas $^{\mathrm{a}}$ & MIALoc $^{\mathrm{a}}$ & HlthLoc & GenLoc $^{\mathrm{b}}$ & FHlth $^{\mathrm{b}}$ & Memory $^{\mathrm{a}}$ \\
\hline Levels & & & & & \\
Age & 0.044 & 0.015 & -0.004 & $0.148^{*}$ & $0.364^{*}$ & $-0.098^{*}$ \\
Sex & -0.226 & $0.373^{*}$ & 0.137 & -0.325 & $3.316^{*}$ & $0.182^{*}$ \\
SRH & 0.192 & -0.135 & $-0.555^{*}$ & $-1.215^{*}$ & $-2.209^{*}$ & $0.331^{*}$ \\
Education & -0.271 & -0.183 & $0.531^{*}$ & -0.372 & $-0.663^{*}$ & $0.239^{*}$ \\
Illnesses & 0.052 & $-0.084^{*}$ & $0.136^{*}$ & -0.087 & $0.410^{*}$ & $0.092^{*}$ \\
\hline Changes & & & & & $0.040^{*}$ & $-0.003^{*}$ \\
Age & 0.011 & 0.007 & -0.002 & 0.010 & -0.031 & -0.003 \\
Sex & 0.130 & -0.022 & -0.033 & $-0.066^{*}$ & $-0.052^{*}$ & 0.011 \\
SRH & $-0.125^{*}$ & 0.009 & 0.027 & 0.023 & $-0.0122^{*}$ & 0.014 \\
Education & $0.247^{*}$ & 0.015 & 0.006 & 0.078 & $0.023^{*}$ & 0.005 \\
Illnesses & $-0.039^{*}$ & 0.008 & 0.007 & $0.018^{*}$ & -0.007 \\
\hline
\end{tabular}

Sex: 0 = male, 1 = female; SRH: 1 = poor to $5=$ excellent; education: left school $\leq 14$ vs. $>14$ years of age; illnesses: number of chronic conditions $(0-60){ }^{*} \mathrm{p}<0.01$.

${ }^{a}$ Higher scores indicate more memory knowledge, internal memory control beliefs, and items recalled.

${ }^{\mathrm{b}}$ Higher scores indicate more external control beliefs and poorer functional health.

memory or if they perceive that their performance is poorer than it has been in the past. Hence, we examined remembering and its correlates in the context of health and other upstream factors, i.e., knowledge of the memory system as well as general and domain-specific control beliefs (about memory and health). The findings move us toward a more nuanced grasp of (a) how aging, subjective beliefs, memory, and health are interconnected. This contextual approach was complemented by examining (b) change over time in each dimension studied, and (c) how individual differences known to affect attrition and performance relate to levels of, and changes in, the target performance and belief dimensions. Findings intimate plausible origins of memory complaints. We will first present key findings regarding static associations among beliefs and performance. We then go on to longitudinal findings and dynamic associations, i.e., correlated change. All effects are a net of covariates and their role is highlighted along the way. For levels, both univariate and multivariate analyses produced significant parameter estimates for means and variances of all dimensions. Most dimensions are interrelated. Behaviorally, better memory and FHlth are associated, concurring with the importance of considering memory and health together [4-6]. Moreover, better outcomes on both indicators are associated with more internal generic beliefs, highlighting the pervasive influence of subjective perceptions on objective performance.

Dynamics of Beliefs, Remembering and Health
Control beliefs themselves are interrelated, extending across domain-specific boundaries. Generic and health control belief orientations are consistent, as are domainspecific memory and health control beliefs. While the first two are modestly associated the latter association is quite strong. The confluence of control beliefs over memory and health, in conjunction with an association between internal health control beliefs and greater knowledge of the memory system, suggests a hitherto unreported crossfertilization of health and memory beliefs. It is feasible that in terms of beliefs, sound memory is considered part of an overall healthy system. In contrast, generic control beliefs and those about memory diverge: holding external generic control beliefs is accompanied by internal beliefs about control over memory. The internalization of responsibility for memory in conjunction with the externalization of generic control may imply a heightened selfregulatory stance in this fundamental cognitive domain that does not apply to lifestyle situations. Finally, and in accord with expectations $[12,13]$, knowledge of the memory system and perceived control over it are inversely related. This suggests that the more informed one is about the workings of memory, the less internal memory control tends to be. This could suggest a sanguine orientation, since many aspects of remembering are not due to personal characteristics, but rather to task or stimulus factors. Yet, this relationship also might suggest a source for SMCs, as people with a poor understanding of the 
memory system may be prone to attributing memory lapses to themselves. The latter view is given some credence by the observed negative relationship between change (decline) in memory knowledge and memory performance. Further research is required to disentangle the costs versus benefits of the inverse relationship be memory knowledge and memory control.

Turning to 12-year trajectories of change, we see significant overall strengthening of internal memory beliefs and external generic control beliefs, in the absence of significant variance in change of these measures, underscoring the ubiquitous nature of these patterns. In contrast, seeming stability in health beliefs and knowledge of the memory system masks the wide individual differences in their trajectories. Likewise, overall health and memory decline emerges from patterns of significant heterogeneity. The behavioral declines themselves are consistent with previous reports $[7,9]$; yet, the heterogeneity in change in both belies considering decline as the norm. We didn't test for lead-lag relationships, but the moderate negative correlation between change in health and memory, is consistent with a report [9] stating that better memory predicts smaller increases in functional limitations, rather than the reverse.

Findings on interrelationships among these patterns of behavioral and belief changes ran contrary to expectations. Neither behavioral decrement was related to changes in its domain-specific control beliefs, nor to generic control, but rather to changes in memory knowledge, which also correlated with changes in domain-specific health beliefs. It is conceivable that the salience (and eventuality) of anticipated memory decline [11] and knowledge of memory tasks work together to bring about SMCs. ALSA respondents, regardless of age, gender, education, and health, attribute to themselves and take responsibility for their memory decline. Knowing how memory works along with this internal orientation provides a background against which memory performance is evaluated, that, in a state of decline, might be consistent with memory complaints, particularly among those with stable or increasing memory knowledge. It may be that as memory knowledge increases, health control beliefs become more internal, and performance suffers. The current series of analyses permit only speculation about possible causal connections among change trajectories; it seems likely that the system is iterative and reciprocal, which our linear analyses cannot test. Mechanisms underlying these relationships are far from clear and a subject for further research. The diversity in patterns of change in memory knowledge may provide one way for- ward, by identifying subgroups of individuals prone to memory complaints.

Inspection of associations between levels and changes shows that poorer health and memory are associated with a more rapid decline in them. Better memory also covaries with improving memory knowledge and FHlth. Turning to relationships with beliefs, having more internal memory beliefs corresponds to more decline in memory knowledge. More internal memory beliefs are associated with less decline in health control, while more external generic control beliefs are associated with more change in health control beliefs. These cross-domain relationships among levels and change in beliefs again underscore a degree of synergy within the control belief system as a whole. This in turn intersects with, and potentially influences, behavioral outcomes. One implication in relation to SMCs is that beliefs about the source of memory success or failure may give rise to negative selfstereotypes, which in turn have a deleterious effect on performance [2].

Although our contextual approach has shed light on a wide array of interconnected systems of beliefs and behavior, it does not provide a complete picture. Most salient is the absence of a measure of SMCs available in the dataset. Hence, we cast the net more widely to explore allied constructs and highlight the basic system in which SMCs may arise. This is important both theoretically and practically if we are to identify the sources and processes underlying memory complaints. Secondly, the study does not explore the role of personality or affect in shaping beliefs, memory, or health, and these factors are likely to play a part $[1,2]$. The nature of the sample and attrition from it constrain generalizations. For instance, all measures commence late in life, so we are unable to identify how preexisting changes color our findings. While the modelling adjusts for missing data, we have not directly examined sample selectivity here. However, other reports [32] suggest that the pattern of findings would be robust.

To our knowledge, this is the first report of individual differences over a protracted period of change in (and relationships among) these particular domains across the span of older adulthood. As such, this work fills an important gap in our understanding of late-life psychological development concerning cross-domain links in memory knowledge, perceptions of control, and both memory and health. Furthermore, examination of informative covariates ensured that the characteristics of our participants were evaluated and their unique effects revealed. Diminishing functional capacity was more marked for those who had left school earlier, judged their health 
more poorly, or reported more chronic illnesses, while only being older adversely affected both health and remembering. Physiological substrates common to both likely play a role $[5,6]$, supporting the theoretical importance of understanding cognitive change within a context that includes health $[2,3,5,6]$. In conclusion, poorer memory and health coexist within a complex system of personal beliefs and knowledge affecting declines in them [14]. Knowledge of the memory system not only appears to play a central role in shaping both recall and attributions of responsibility for it, but also may give rise to SMCs if there is a dissociation between one's understanding of the memory system and proficiency as a remember. The origins and veracity of memory complaints are likely to remain elusive until we better understand the interplay of subjective beliefs and the crucial domains of cognitive and physical integrity.

\section{Acknowledgements}

We would like to thank the participants in the ALSA who have given their time over many years, and without whom the present study would not have been possible. The ALSA was supported by grants from the South Australian Health Commission, the Australian Rotary Health Research Fund, the US National Institute on Aging (Grant No. AG 08523-02), the Office for the Aging (S.A.), Elderly Citizens Homes (S.A.), the National Health and Medical Research Council (NH\&MRC 22922), the Premiers Science Research Fund (S.A.) and the Australian Research Council (DP0879152; DP130100428). The authors gratefully acknowledge the work of the project team and of Deputy Director Tim Windsor at the Flinders Centre for Ageing Studies, Flinders University, who assisted with various aspects of the ALSA.

\section{References}

1 Hertzog C, Pearman A: Memory complaints in adulthood and old age; in Perfect TJ, Lindsay DS (eds): The SAGE Handbook of Applied Memory. London, Sage Publications, 2013, pp 423-443.

2 Hess TM: Memory and aging in context. Psychol Bull 2005;131:383-406.

3 Luszcz MA: Memory and ageing: Cognitive and functional approaches; in Heaven P (ed): Psychological Bases of Human Development. Sydney, Harcourt Brace Jovanovich, 1992, pp 302-328.

4 Agrigoroaei S, Lachman ME: Cognitive functioning in midlife and old age: combined effects of psychosocial and behavioral factors. J Gerontol B Psychol Sci Soc Sci 2011;66(suppl 1):i130-i140.

5 Infurna F, Gerstorf D: Linking perceived control, physical activity, and biological health to memory change. Psychol Aging 2013;28: 1147-1163.

6 Spiro A 3rd, Brady CB: Integrating health into cognitive aging: toward a preventive cognitive neuroscience of aging. J Gerontol B Psychol Sci Soc Sci 2011;66(suppl 1):i17-i25.

7 Anstey KJ, Hofer SM, Luszcz MA: A latent growth curve analysis of late-life sensory and cognitive function over 8 years: evidence for specific and common factors underlying change. Psychol Aging 2003;18:714-726.

8 Gerstorf D, Ram N: Limitations on the importance of self-regulation in old age. Hum Dev 2009;52:38-43.

-9 Infurna FJ, Gerstorf D, Ryan LH, Smith J: Dynamic links between memory and functional limitations in old age: longitudinal evidence for age-based structural dynamics from the AHEAD study. Psychol Aging 2011;26:546548.

Dynamics of Beliefs, Remembering and Health
10 Lachman ME, Neupert SD, Agrigoroaei S: The relevance of control beliefs for health and aging; in Schaie KW, Sherry LW (eds): Handbook of the Psychology of Aging, ed 7. San Diego, Academic Press, 2011, pp 175-190.

11 Skinner EA: Perceived Control, Motivation, and Coping: Individual Differences and Development Series. Thousand Oaks, 1995, vol 8.

12 Hertzog C, Dixon RA, Schulenberg JE, Hultsch DF: On the differentiation of memory beliefs from memory knowledge: the factor structure of the Metamemory in Adulthood scale. Exp Aging Res 1987;13:101-107.

13 Luszcz M: When knowing is not enough: the role of memory beliefs in prose recall of older and younger adults. Aust Psychol 1993;28: 16-20.

14 Baltes PB, Lindenberger U, Staudinger U: Life-span theory in developmental psychology; in Lerner RM (ed): Handbook of Child Psychology, ed 6. New York, Wiley, 2006, vol 1: Theoretical Models of Human Development, pp 559-664.

15 Luszcz M, Bryan J, Kent P: Predicting episodic memory performance of very old men and women: contributions from age, depression, activity, cognitive ability and speed. Psychol Aging 1997;12:340-351.

16 Lachman ME, Rosnick CB, Röcke C: The rise and fall of control beliefs in adulthood: cognitive and biopsychosocial antecedents and consequences of stability and change over nine years; in Bosworth HB, Hertzog C (eds): Aging and Cognition: Research Methodologies and Empirical Advances. Washington, APA Press, 2009, pp 143-460.
7 Lachman MA, Weaver SL: Sociodemographic variations in the sense of control by domain: findings from the MacArthur studies of midlife. Psychol Aging 1998;13:553-562.

18 Wallston K, Wallston B: Health locus of control scales; in Lefcourt HM (ed): Research with the Locus of Control Construct: Assessment Methods. New York, Academic Press, 1981, pp 189-244.

19 Fry PS, DeBats D: Sources of human lifestrengths, resilience, and health; in Fry PS, Keyes DJL (eds): New Frontiers in Resilient Aging: Life-Strengths and Well-Being in Late Life. Cambridge, Cambridge University Press, 2010, pp 15-59.

20 Lachman ME: Locus of control in aging research: a case for multidimensional and domain-specific assessment. Psychol Aging 1986;1:34-40.

21 Lachman ME: Perceived control over agingrelated declines: adaptive beliefs and behaviours. Curr Dir Psychol Sci 2006;15:282-286.

22 Windsor T, Anstey K: A longitudinal investigation of perceived control and cognitive performance in young, midlife and older adults. Neuropsychol Dev Cogn B Aging Neuropsychol Cogn 2008;15:744-763.

23 Valentijn SAM, Hill RD, Van Hooren SAH, Bosma H, Van Boxtel MPJ, Jolles J, et al: Memory self-efficacy predicts memory performance: results from a 6-year follow-up study. Psychol Aging 2006;21:165-172.

24 Anstey KJ, Luszcz MA, Andrews G: Psychosocial factors, gender and late-life mortality. Ageing Int 2002;27:73-89.

25 Infurna FJ, Gerstorf D, Ram N, Schupp J, Wagner GG: Long-term antecedents and outcomes of perceived control. Psychol Aging 2011;26:559-575. 
26 Gatz M, Karel MJ: Individual change in perceived control over 20 years. Int J Behav Dev 1993; 16:305-322.

-27 Mirowsky J, Ross C: Life course trajectories of perceived control and their relationship to education. Am J Sociol 2007;112:1339-1382.

28 Gerstorf D, Röcke C, Lachman ME: Antecedent-consequent relations of perceived control to health and social support: longitudinal evidence for between-domain associations across adulthood. J Gerontol B Psychol Sci Soc Sci 2011;66:61-71.

29 Ross CE, Mirowsky J: Age and the gender gap in the sense of personal control. Soc Psychol Q 2002;65:125-145.

30 Luszcz MA: Beliefs about control in later life: implications of perceptions of health, memory and global control. Hong Kong J Gerontol 1996; 10:502-506.
1 Andrews GR, Clark MS, Luszcz MA: Successful aging in the Australian Longitudinal Study of Aging: applying the MacArthur Model cross-nationally. J Soc Issues 2002;58:749765.

32 Luszcz MA, Giles L, Anstey KJ, Browne-Yung K, Walker R, Windsor TD: Cohort profile: the Australian Longitudinal Study of Ageing (ALSA). Int J Epidemiol, in press.

33 Dixon RA, Hertzog C, Hultsch DF: The multiple relationships among Metamemory in Adulthood (MIA) scales and cognitive abilities in adulthood. Hum Learn J Practical Res Appl 1986;5:165-177.
34 Reid DW, Ziegler M: Validity and stability of a new desired control measure pertaining to psychological adjustment of the elderly. J Gerontol 1980;35:395-402.

35 Rosow I, Breslau N: A Guttman health scale for the aged. J Gerontol 1966;21:556-559.

36 Nagi SZ: An epidemiology of disability among adults in the United States. Milbank Mem Fund Q Health Soc 1976;54:439-467.

37 Schafer JL, Graham JW: Missing data: our view of the state of the art. Psychol Methods 2002;7:147-177.

38 McArdle JJ: Latent growth within behavior genetic models. Behav Genet 1986;16:163200.

39 Meredith W, Tisak J: Latent curve analysis. Psychometrika 1990;55:107-122.

40 Muthén L, Muthén B: Mplus User's Guide. Los Angeles, Muthén \& Muthén, 1998, vol 6. 